\title{
Modelación Numérica y Mapas de Afectación por Flujo de Lahares Primarios en el Drenaje Sur del Volcán Cotopaxi
}

\author{
Vera, Pablo $^{1}$; Ortega, Patricio ${ }^{1}$; Casa, Edwin ${ }^{1}$; Santamaría, Jorge ${ }^{1}$; Hidalgo, Ximena ${ }^{1}$ \\ ${ }^{1}$ Escuela Politécnica Nacional, Facultad de Ingeniería Civil, Centro de Investigaciones y Estudios en Recursos Hídricos \\ CIERHI, Quito, Ecuador
}

\begin{abstract}
Resumen: Los lahares primarios originados durante erupciones de volcanes nevados, como el volcán Cotopaxi, son el resultado de la combinación de mecanismos físicos relacionados con el fenómeno eruptivo como la expulsión de ceniza, material piroclástico y flujos de lava incandescente que provocan el derretimiento súbito de una porción del glaciar. Afectan directamente asentamientos humanos e infraestructura desarrollada a lo largo de los cauces de los ríos y llanuras que corresponden a los drenajes naturales por donde transitan los lahares. El periodo de recurrencia eruptiva del volcán es relativamente amplio considerando la más reciente erupción significativa que ha sido registrada en junio de 1877. La investigación se enfoca en la modelación numérica unidimensional para flujo no permanente realizada en el programa libre HEC-RAS, considerando información geológica, glaciológica, vulcanológica y cartográfica actual, generada y recopilada en campo durante los últimos años. Estos datos han sido analizados y considerados para la definición de los parámetros iniciales que corresponden a volúmenes e hidrogramas. El modelo numérico calibrado en base al evento histórico de 1877, constituye la base para la simulación de los escenarios probables de ocurrencia. Los resultados obtenidos permiten la generación de mapas de afectación referenciales que constituyen un aporte técnico y práctico, ya que pueden ser utilizados para tomar decisiones acerca de la definición de zonas de afectación, sitios seguros, planificación territorial, planes de concientización, recuperación y mitigación ante procesos eruptivos futuros del volcán Cotopaxi que afecten de manera particular el valle de Latacunga.
\end{abstract}

Palabras clave: Modelación Numérica, Lahares, Mapas, Cotopaxi.

\section{Numerical Model and Maps by Primary Lahars in Southern Drainage of Cotopaxi Volcano}

\begin{abstract}
Primary lahar flows are originated during eruptions of ice-capped volcanoes, such as the Cotopaxi volcano, as the result of the combination of physical mechanisms related to eruptive phenomenon such as the expulsion and fall of ash, pyroclastic material and incandescent lava flows that can cause sudden melting of a portion of the glacier. Lahars have a direct incidence on human settlements including infrastructure built along the rivers and plains that correspond to the natural drainages through which the laharic flows pass. Cotopaxi eruption recurrence is relatively long given that the last significant event was recorded in June 1877. The overall purpose of the study is to present the one-dimensional numerical simulation results for non-permanent flow employing the open-source software HECRAS. The boundary conditions as DEM, melt volumes, hydrographs among others was the result of up-to-date geological, glaciological, volcanological and cartographic information, generated and compiled during the last years. The calibrated model based on 1877 historical event is the cornerstone for the numerical modelling of the probable scenarios. The obtained results allow the generation of referential maps which are a practical contribution since they can be used to make decisions regarding the affected areas, safe sites, territorial planning, awareness, recovery and mitigation plans in the presence of the Cotopaxi eruption with particular incidence in Latacunga Valley.
\end{abstract}

Keywords: Numerical Modeling, Lahars, Maps, Cotopaxi.

\section{INTRODUCCIÓN}

El crecimiento de zonas urbanas y rurales, así como el desarrollo industrial e infraestructura pública y privada en el sector del valle de Latacunga, provincia de Cotopaxi, presenta relación directa frente a la vulnerabilidad asociada a los flujos de lahar generados durante una posible erupción del volcán Cotopaxi. Luego de un período relativamente largo de inactividad, más de 140 años desde la última erupción representativa (Sodiro, 1877; Wolf, 1878), durante el último trimestre del año 2015 e inicios de 2016 se registró un nuevo periodo de reactivación de la actividad volcánica (IG-EPN, 2015). Ante este proceso natural que podría afectar negativamente extensas zonas ubicadas en sectores próximos a los cauces naturales de drenaje del flujo de lahar, se requiere información técnica actualizada relacionada con la ocurrencia de este fenómeno para contribuir al estudio y problemática particular que representa el flujo de lahares primarios producto de erupciones volcánicas en volcanes nevados. 
Para alcanzar este objetivo, se hace evidente la necesidad de llevar a cabo actividades concernientes a la recopilación y levantamiento de información de campo correspondiente principalmente a vestigios o evidencias físicas asociadas al evento eruptivo ocurrido en junio de 1877. Las actividades técnicas desarrolladas en las últimas décadas por el Instituto Geofísico de la Escuela Politécnica Nacional (IG-EPN), en varias campañas han permitido disponer de registros de campo sobre la caracterización geológica de los vestigios del evento eruptivo del año 1877 en varias secciones transversales representativas de la zona de estudio. Además, con base en el análisis de las muestras recuperadas, se ha caracterizado el tipo de lahar y su composición (Mothes et al., 2004; Mothes, 2006; Hall et al., 2008).

El avance tecnológico en relación a la generación de modelos digitales de terreno (MDT), que en el caso de esta investigación alcanza una resolución espacial de 3.0 metros por pixel, permite la representación adecuada de la topografía en el área de estudio. Esta información es utilizada para la modelación numérica del flujo de lahar empleando un paquete computacional de código libre para el análisis de flujo permanente y no permanente (no estacionario) que resuelve las ecuaciones de Saint Venant presentadas en forma de derivadas parciales en una dimensión, en la dirección del flujo de un volumen de control considerando los principios de conservación de masa y cantidad de movimiento (Vera, 2017).

El análisis numérico del flujo de lahares del volcán Cotopaxi ha sido ampliamente estudiado desde hace varias décadas tomando como referencia al evento eruptivo histórico del año 1877, considerando en ciertos casos, modelos numéricos sencillos asumiendo flujos homogéneos con volumen constante (Barberi et al., 1992). En lo reportado por Castro et al., 1992; Espín, 1993 y Sánchez, 1993, la modelación numérica de lahares del Cotopaxi se realizó con programas computacionales como el HEC-1, LAHAR-1 y DAMBRK (Costa, 1977) respectivamente. Se ha investigado los lahares y su impacto sobre ciudades como Latacunga con el programa TITAN2D (Williams, 2006). Según lo reportado por Lima, 2007, se realiza la simulación del flujo lahárico no permanente utilizando programas como el FLDWAV y HEC-RAS. En los estudios desarrollados por Samaniego, 2004; Pistolesi, 2014, se estudia los flujos de lahar mediante el uso del software LAHARZ (Iverson et al., 1998).

En varios casos, las condiciones iniciales de las simulaciones consideran la evidencia histórica geológica encontrada en los trabajos de campo como base de la modelación numérica e información topográfica correspondiente a modelos digitales de terreno para la representación del área de estudio.

\section{EL VOLCÁN COTOPAXI}

Es un estratovolcán ubicado en la cordillera Oriental o Real de los Andes Ecuatorianos, a $60 \mathrm{~km}$ al Sureste de Quito, su cráter se eleva hasta los 5897 m.s.n.m. El cono del volcán se encuentra cubierto por un casquete glaciar que desciende desde la cima por todos los flancos del mismo.

La aparente uniformidad de su topografía se interrumpe ante la presencia de varias quebradas profundas, que constituyen el inicio o nacimiento de los cauces principales y ríos, los mismos que fluyen aguas abajo atravesando el valle de Latacunga en el drenaje Sur-Occidental, el valle de los Chillos hacia el Norte y además hacia el drenaje Oriental.

Desde mediados del mes de abril del año 2015, el IG-EPN reportó un incremento en la actividad eruptiva del volcán, alcanzándose un total de 300 sismos volcánicos y varias emisiones de dióxido de azufre (SO2) que precedieron a explosiones hidromagmáticas (Gaunt et al., 2016). La actividad superficial caracterizada por emisión de gas y ceniza continuó durante meses, registrándose un volumen de 860 mil metros cúbicos de ceniza (Bernard et al., 2016). Las características de este periodo eruptivo han permitido identificar a este evento con el Índice de Explosividad Volcánica VEI igual a 1 (VEI; Newhall \& Self, 1982).

Según lo reportado por Jordan (1983), el área total del casquete glaciar cubría $21.3 \mathrm{~km}^{2}$ en el año 1976. Esta cobertura glaciar ha sufrido una reducción notable que continúa a través de los años, alcanzando valores totales de hasta $12.7 \mathrm{~km}^{2}$ en el año 2006 y aproximadamente $10.5 \mathrm{~km}^{2}$ para el año 2016 (Cáceres, 2016). Por analogía con la erupción del año 1995 del Nevado del Ruiz se estima una disminución en el espesor del glaciar de alrededor de 4 metros. Dado que el agua se libera de forma súbita el hidrograma tipo de estos flujos tendría una forma triangular, con un caudal dominante y una atenuación lenta con hidrogramas de iguales características a los utilizados para modelar los eventos laháricos de las erupciones del Mount. St. Helens y del Nevado del Ruiz (Pierson et al., 1990; Thouret, 1990) respectivamente.

Samaniego (2004) y Andrade et al. (2005) definieron escenarios eruptivos ante una posible erupción del volcán Cotopaxi en base a criterios como: Dinamismo eruptivo (tipos de erupciones que podrían ocurrir en el volcán), magnitud de las erupciones (establecida por trabajos de campo y distribución de los productos volcánicos), comportamiento de otros volcanes andesíticos (Tungurahua, Reventador y Sangay), grado de interacción entre los productos volcánicos y el glaciar (función de los dinamismos eruptivos), tamaño y porcentaje de fusión del glaciar.

En base a las características eruptivas de cada uno de los ciclos de actividad categorizados según el Índice de Explosividad Volcánica (VEI; Newhall \& Self, 1982), se ha establecido, para el volcán Cotopaxi, cuatro escenarios que permiten determinar la probabilidad de ocurrencia de una erupción volcánica. Considerando aspectos como el índice de explosividad volcánica VEI, el tipo de actividad y los fenómenos eruptivos como la caída de ceniza, flujos piroclásticos, fragmentos balísticos y lavas, se determinan los siguientes escenarios eruptivos: Pequeño (Escenario 1: VEI 12), Moderado (Escenario 2: VEI 2-3), Grande (Escenario 3: VEI 3-4) y Muy grande (Escenario 4: VEI >4).

En los últimos 2000 años se han registrado al menos 19 eventos eruptivos importantes, el más reciente se ha catalogado con VEI igual a 1, se registra otros 6 eventos eruptivos con un VEI de 3 (se incluye un evento con un VEI de 2-3), adicionalmente se consideran 10 eventos con un VEI de 4 (se incluye un evento con un VEI de 3-4), y finalmente hay 2 eventos eruptivos con un VEI > 4 (Samaniego, 2004). 
La secuencia cronológica presentada en la Tabla 1 permite plantear que el escenario con mayor probabilidad de ocurrencia es el Escenario No. 3, el cual está asociado a una erupción similar a la ocurrida en el año 1877 (VEI 3-4) asignándole un $58 \%$ de probabilidad. Según Samaniego (2004), luego de un ciclo de reposo relativamente extenso como el actual (más de 140 años), existe la posibilidad de que la actividad del volcán continúe y desencadene una erupción importante (VEI $\geq 3$ ). Para los eventos mayores (Escenario 4: VEI >4) la probabilidad de ocurrencia alcanza el $10.5 \%$, mientras que los eventos menores (Escenario 2: VEI 2-3) presentan una probabilidad del $26.31 \%$. La probabilidad de que un evento eruptivo produzca únicamente un flujo de lava es relativamente baja ( 2 erupciones en los últimos 2000 años, con probabilidad de $10.52 \%$ ).

Tabla 1. Síntesis de la actividad volcánica del Cotopaxi en los últimos 2000 años. Samaniego (2004)

\begin{tabular}{|c|c|c|c|c|c|}
\hline Edad & $\begin{array}{c}\text { Emisiones de } \\
\text { ceniza }\end{array}$ & $\begin{array}{c}\text { Flujo } \\
\text { piroclástico }\end{array}$ & Lahares & $\begin{array}{c}\text { Flujo de } \\
\text { lava }\end{array}$ & VEI \\
\hline $2015 \mathrm{DC}$ & $\mathrm{x}$ & & secundario & & 1 \\
\hline $1880 \mathrm{DC}$ & $\mathrm{x}$ & & & & $2-3$ \\
\hline $1877 \mathrm{DC}$ & $\mathrm{x}$ & $\mathrm{x}$ & 2 & & 4 \\
\hline $1854 \mathrm{DC}$ & $\mathrm{x}$ & $\mathrm{x}$ & 1 & 1 & 3 \\
\hline $1853 \mathrm{DC}$ & $\mathrm{x}$ & & 1 & 1 & 3 \\
\hline $1768 \mathrm{DC}$ & $\mathrm{x}$ & $\mathrm{x}$ & 1 & 1 & 4 \\
\hline $1766 \mathrm{DC}$ & $\mathrm{x}$ & $\mathrm{x}$ & 1 & & 3 \\
\hline $1744 \mathrm{DC}$ & $\mathrm{x}$ & $\mathrm{x}$ & 2 & & 4 \\
\hline $1742 \mathrm{DC}$ & $\mathrm{x}$ & $\mathrm{x}$ & 3 & & 4 \\
\hline $1532-34 \mathrm{DC}$ & $\mathrm{x}$ & $\mathrm{x}$ & 2 & 1 & 4 \\
\hline$\sim 1150 \mathrm{DC}$ & $\mathrm{x}$ & $\mathrm{x}$ & 2 & & 4 \\
\hline$\sim 1000 \mathrm{DC}$ & $\mathrm{x}$ & $\mathrm{x}$ & & & 3 \\
\hline $950 \mathrm{DC}$ & $\mathrm{x}$ & $\mathrm{x}$ & 2 & & $>4$ \\
\hline $850 \mathrm{DC}$ & $\mathrm{x}$ & $\mathrm{x}$ & 1 & & $3-4$ \\
\hline $750 \mathrm{DC}$ & $\mathrm{x}$ & $\mathrm{x}$ & 2 & & 4 \\
\hline$\sim 180 \mathrm{DC}$ & $\mathrm{x}$ & $\mathrm{x}$ & 1 & varios & 4 \\
\hline$\sim 150 \mathrm{DC}$ & $\mathrm{x}$ & $\mathrm{x}$ & 2 & 1 & $>4$ \\
\hline$\sim 100 \mathrm{DC}$ & $\mathrm{x}$ & & varios & & 4 \\
\hline $50 \mathrm{AC}$ & $\mathrm{x}$ & $\mathrm{x}$ & & & 4 \\
\hline
\end{tabular}

\section{LA RED DE DRENAJE SUR}

El entorno natural del área estudiada corresponde al drenaje Sur-Occidental que inicia en las faldas del volcán, donde el sistema de quebradas transporta, desde las once (11) lenguas de glaciar, los flujos de lahares hasta cauces más amplios y menos profundos (valle de Latacunga) con una longitud de 30 km que conforman el río Cutuchi, el cual atraviesa la ciudad y continua hacia el Sur donde presenta una morfología de cauce encañonado y profundo.

La red hidrográfica primaria del flanco Sur-Occidental está compuesta por los ríos Cutuchi, Saquimala y Aláquez. Estos cauces de drenaje natural fluyen desde la base del volcán y continúan por una llanura extensa de baja pendiente hacia el Sur y el Occidente. En el caso particular del río Pumacunchi, que pertenece a la cordillera Occidental, se observa que al desarrollarse en forma paralela y cercana al cauce del río Cutuchi puede ser afectado en el caso del desborde del lahar.
Se consideran cuatro tramos iniciales para representar la morfología alta o superior de la zona estudiada, cuyos nombres son: Cutuchi, San Lorenzo, Pucahuaicu y Aláquez. Conforme se desarrollan hacia el Sur-Occidente forman los tramos intermedios del río Cutuchi y Saquimala, que al unirse con el río Aláquez, conforman el tramo final del río Cutuchi. Las pendientes longitudinales representativas de los tramos considerados varían entre 1 y $10 \%$ y las longitudes de los cauces alcanzan valores desde 9 hasta $42 \mathrm{~km}$.

La distribución radial del glaciar del Cotopaxi reportada por Cáceres (2016), referente a las áreas de aporte del drenaje Sur, permite identificar las lenguas glaciares, que se han denominado y numerado de acuerdo a lo siguiente: Tambo (9), Churrumihurco (10), Simarrones (11), El Picacho (12), Saquimala (13), San Lorenzo (14), Capuli-Huaicu (15), Pucahuaicu Oeste (16), Chanchunga Sur (17), Chanchunga Norte (18) y Yanasacha (19).

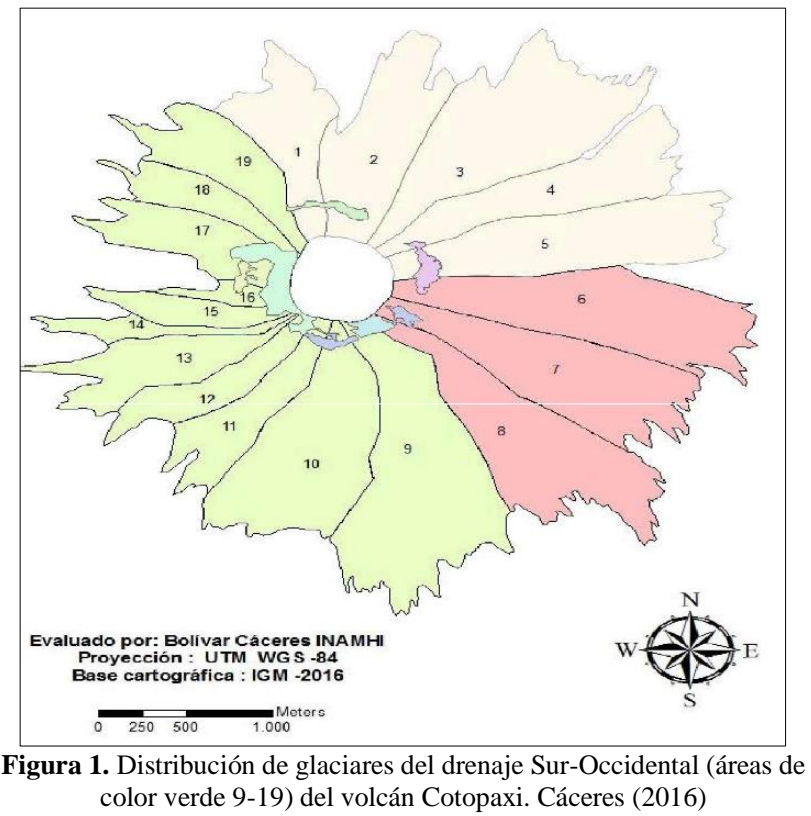

En la Tabla 2 se presentan las áreas glaciares de drenaje entre los años 1977 y 2016 (Cáceres 2017), correspondientes a la zona Sur-Occidental. Los resultados obtenidos muestran la notable reducción de aproximadamente el $50 \%$ en la superficie glaciar, comparando las áreas totales de los años 1977 y 2016.

Tabla 2. Evolución de áreas glaciares del flanco Sur-Occidental entre los

\begin{tabular}{cccc}
\multicolumn{4}{c}{ años 1977 y 2016. Cáceres (2017) } \\
\hline$\#$ & Glaciar & $\begin{array}{c}\text { Área 1977 } \\
\left(\mathbf{m}^{\mathbf{2}}\right)\end{array}$ & $\begin{array}{c}\text { Área 2016 } \\
\left(\mathbf{m}^{2} \mathbf{)}\right.\end{array}$ \\
\hline 9 & Tambo & 893101 & $1^{\prime} 183565$ \\
10 & Churrumihurco & $1^{\prime} 348900$ & 894343 \\
11 & Simarrones & $1^{\prime} 585600$ & 362736 \\
12 & El Picacho & $1^{\prime}$ '403 500 & 275678 \\
13 & Saquimala & $1^{\prime} 177174$ & 402897 \\
14 & San Lorenzo & $1^{\prime} 192300$ & 137166 \\
15 & Capuli-Huaicu & 776100 & 180454 \\
16 & Pucahuaicu Oeste & 426872 & 62377 \\
17 & Chanchunga Sur & 382600 & 325413 \\
18 & Chanchunga Norte & 712300 & 239722 \\
19 & Yanasacha & 957400 & 398034 \\
& Área Total & $\mathbf{1 0} \mathbf{8 5 5} \mathbf{8 4 7}$ & $\mathbf{4} \mathbf{4 6 2} \mathbf{4 8 5}$ \\
\hline
\end{tabular}


Cabe señalar que en la etapa de calibración del modelo numérico se utiliza la información correspondiente al área del glaciar del año 1977, puesto que constituye la mejor aproximación al evento del año 1877 (VEI 3-4) debido a su elevada probabilidad de ocurrencia. Para la modelación numérica de los escenarios eruptivos actuales se emplea las áreas de glaciar en las condiciones actuales, las mismas que como se ha determinado, son relativamente menores a las del escenario de calibración (evento eruptivo histórico).

\section{ANÁLISIS TEÓRICO / METODOLOGÍA}

\subsection{Breve caracterización reológica de lahares}

Un lahar se define como una mezcla de sólidos y agua que fluye rápidamente por efecto de la gravedad. Está compuesto por bloques de roca, escombros, sedimentos y agua. Sus características pueden variar conforme transcurre el tiempo y la distancia que recorre hacia aguas abajo desde el inicio del evento en el pie del volcán. Entre los tipos de flujo que se presentan tenemos a los flujos de escombros, transicionales, hiperconcentrados y las inundaciones (Vera, 2017).

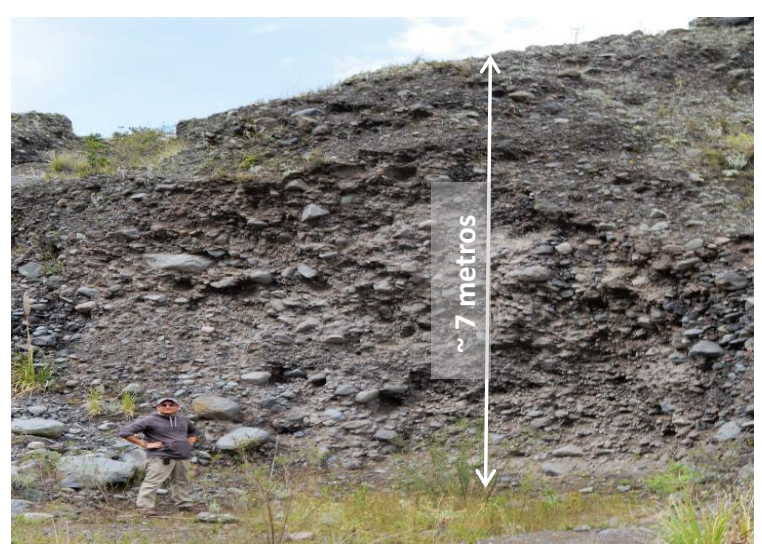

Figura 2. Depósito de lahar primario en el río Aláquez. Evento eruptivo del año 1877. Vera (2017)

Los factores que caracterizan un flujo lahárico son: ocurrencia repentina relacionada con el tipo de erupción, elevada tasa de avance que depende de las pendientes y la topografía, gran capacidad de transporte de sólidos que se van incorporando durante su recorrido, desarrollo en grandes distancias a lo largo de los cauces naturales y zonas inundables que generalmente albergan a poblaciones e infraestructura.

Los flujos de lahares alcanzan caudales máximos en tiempos relativamente cortos, erosionan cauces e incorporan material sedimentario que corresponde a depósitos de lahar existentes sobre el terreno originados por eventos anteriores. El volumen de agua presente en el lahar genera cantidades de material que pueden superar la capacidad hidráulica de los drenajes y provocan inundaciones y desbordamientos en zonas planas.

Según Coussot y Meunier (1995) los tipos de flujo se clasifican de manera conceptual y cualitativa en función del porcentaje de sólidos que componen la mezcla, la interacción de las partículas (cohesivas, no cohesivas, granulares, finos, etc.) y la fracción de sólidos (cuyo valor se incrementa desde agua a flujos con transporte de sólidos, flujos hiperconcentrados, flujos de escombros y avalanchas).

En la Figura 3 se presenta una clasificación en función de la fracción de sólidos y el tipo de material, de acuerdo a este esquema se puede clasificar a los lahares como una mezcla bifásica con movimiento rápido, que se encuentra entre los flujos de escombros (desperdicios) e hiperconcentrados.

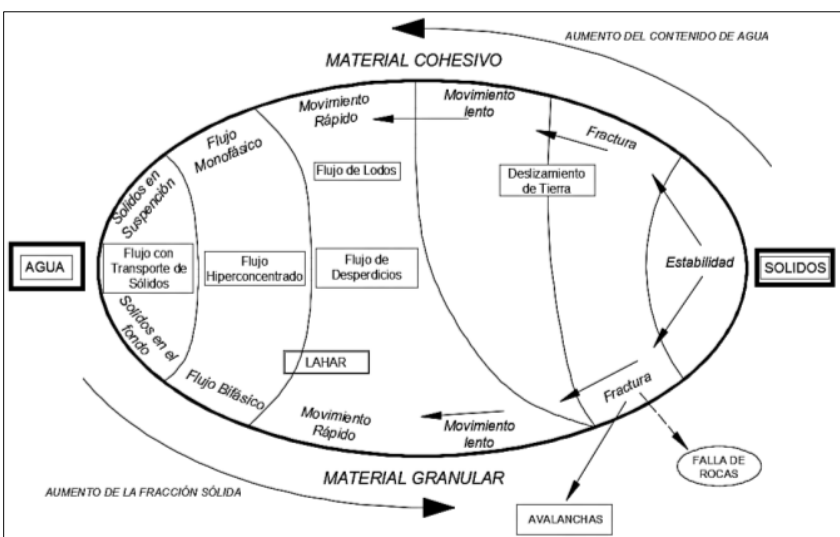

Figura 3. Clasificación de las mezclas de agua y sólido como función de la fracción de sólidos y el tipo de material. Coussot y Meunier (1995)

Estudios realizados en el drenaje Sur del volcán Cotopaxi permiten determinar las características físicas de los flujos de lahar de esta zona. Según Mothes et al. (2004), estos presentan características de flujos no cohesivos e hiperconcentrados con distribución casi uniforme de los granos que conforman la matriz del flujo. Esto se ratifica en estudios más recientes (CIERHI, 2017) que analizan la distribución del tamaño del grano en función del porcentaje acumulado de la muestra donde se observa un comportamiento como flujo granular y no cohesivo al clasificarlo en función del tamaño medio del grano y el sorteo representado como la variación de la muestra en relación a su valor promedio en cada sección.

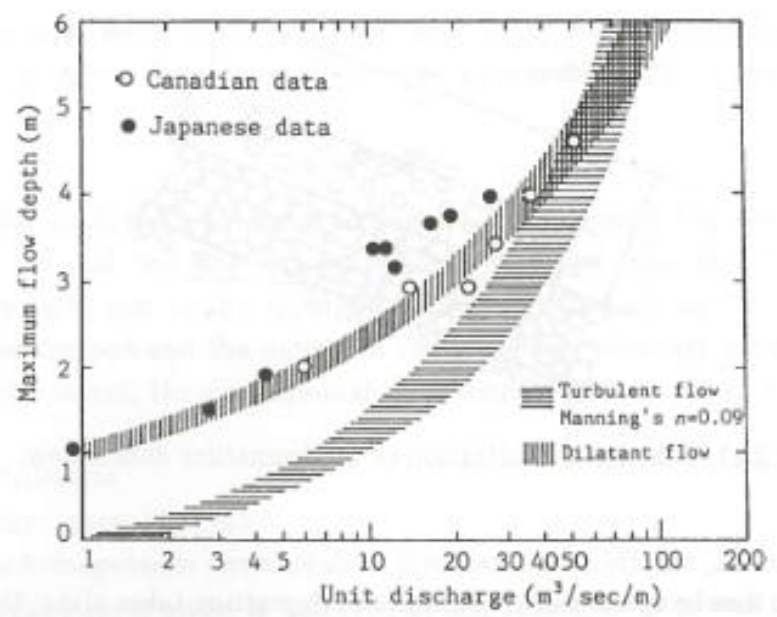

Figura 4. Comparación de caudales unitarios obtenidos para diferentes profundidades con flujos de escombros granulares. Takahashi (1991)

En la investigación experimental realizada por Takahashi (1991) se plantea que el comportamiento, tanto de un flujo dilatante (lahar) como de uno turbulento, tiende a ser similar conforme aumenta el valor de caudal unitario en relación al incremento de la profundidad de flujo. Esta observación 
permite plantear métodos de solución numérica de flujos laháricos como flujos Newtonianos, puesto que generalmente se consideran calados y caudales unitarios elevados.

\subsection{Coeficientes de rugosidad (resistencia al movimiento)}

Los coeficientes de rugosidad o “n” de Manning están relacionados con la resistencia al movimiento como características del cauce que generalmente están asociadas a la rugosidad superficial, presencia de vegetación, la forma y alineación del cauce.

La ecuación de Manning (Ganguillet y Kutter, 1889) involucra el término del coeficiente de rugosidad (n), el mismo que según lo reportado por Chow (1994) se expresa como:

$$
V=\frac{1}{n} R^{2 / 3} S^{1 / 2}
$$

Donde $V$ es la velocidad media en $(\mathrm{m} / \mathrm{s}), n$ el coeficiente de rugosidad o " $n$ " de Manning, $R$ es el radio hidráulico en (m) y $S$ es la pendiente de la línea de energía $(\mathrm{m} / \mathrm{m})$.

Para Costa (1997), los flujos ricos en sedimentos o hiperconcentrados, pueden ser modelados utilizando coeficientes de resistencia al movimiento correspondientes a flujos dilatantes, sin embargo, requieren de una etapa de calibración que permita un análisis entre los resultados alcanzados y los datos de campo que generalmente están constituidos por vestigios de eventos pasados.

\subsection{Modelación numérica unidimensional}

La modelación numérica unidimensional se basa en la solución de las ecuaciones que describen el comportamiento de un flujo y cuyas características más relevantes son el caudal, velocidad, calado, resistencia al movimiento, entre otras. El modelo considerado para la simulación numérica se utiliza para las etapas de calibración y modelación unidimensional definitiva para flujos permanentes y no permanentes.

El modelo numérico unidimensional utilizado es HEC-RAS 5.0.3 de la U.S. Army Corps of Engineers, permite simular flujo uniforme gradualmente variado mediante la solución de la ecuación de la energía, donde las pérdidas se evalúan por fricción con la ecuación de Manning y tanto la expansión como la contracción en función de coeficientes multiplicados por las cargas de velocidad.

La ecuación de impulso o cantidad de movimiento es utilizada cuando el perfil de flujo es rápidamente variado. Las ecuaciones de continuidad y conservación de la cantidad de movimiento para flujos unidimensionales en canales abiertos se pueden expresar como se plantea a continuación (HECRAS, 2016):

\section{Ecuación de Continuidad:}

$$
\frac{d A}{d t}+\frac{d Q}{d x}-q_{l}=0
$$

\section{Ecuación de Cantidad de movimiento:}

$$
\frac{\partial Q}{\partial t}+\frac{\partial Q V}{\partial x}+g A\left(\frac{\partial z}{\partial x}+S_{f}\right)=0
$$

Donde $A$ es la sección transversal, $t$ es el tiempo, $Q$ es el caudal, $x$ representa la distancia a lo largo del canal, $q_{l}$ es el caudal lateral por unidad de longitud, $V$ representa la velocidad, $g$ es la aceleración de la gravedad, $z$ representa el nivel de referencia del canal, $S_{f}$ es la pendiente de fricción.

La simulación numérica para flujo no permanente requiere la solución de las ecuaciones de Saint Venant. La necesidad de encontrar la solución a estas ecuaciones hizo que se desarrollen métodos simplificados que al despreciar algunas de las variables hidrodinámicas menos preponderantes permiten encontrar la solución numérica. Entre los métodos de resolución se encuentran los métodos hidrológicos, de onda cinemática, onda difusiva, onda dinámica cuasi-permanente. En general los métodos de resolución simplificados se utilizan para modelación hidrológica, ya que las limitaciones en cuanto a capacidad y velocidad de los procesadores actualmente permiten obtener resultados con un gasto computacional reducido y en tiempos relativamente cortos.

El programa HEC-RAS 5.0.3, emplea un método que no considera la viscosidad artificial y se denomina "Local Partial Inertia ( $L P I)$ ". Este método consiste en añadir un término a las ecuaciones para reducir los términos de inercia cuando el número de Froude se acerca a la unidad, donde se producirá invariablemente un cambio de régimen en el flujo y se genere una posible discontinuidad. En el método LPI se incluye el factor "Exponent for Froude number reduction factor" conocido como factor " $m$ ". Cuando este valor es igual a la unidad se consigue resultados estables. Un factor de ponderación que se aplica a las ecuaciones en diferencias finitas usadas en el cálculo del régimen variable es el "Theta Weighting Factor", cuando este valor es cercano a 1.0, se alcanza mayor estabilidad, sin embargo, valores alrededor de 0.6 permitirán disponer de resultados más precisos al realizar la simulación numérica (Hamad, 2008).

Existen varios factores que intervienen en la estabilidad del modelo numérico entre los que se destacan los siguientes:

- La distancia entre secciones transversales, puesto que a menor distancia entre ellas se alcanza mayor estabilidad con una mayor demanda del tiempo de cálculo.

- El intervalo de cálculo (time-step), produciéndose mayor estabilidad numérica a medida que este valor es pequeño a cambio de un elevado tiempo de simulación.

- La simulación previa en régimen permanente es una aproximación a la solución final que permite alcanzar estabilidad en el modelo con para flujo no permanente.

\subsection{Definición de hidrogramas de crecida}

Según Samaniego (2004), dada la duración del evento eruptivo, el hidrograma típico o característico para un lahar inducido por un flujo de lava tendría un inicio lento y 
probablemente varios pulsos. La erupción volcánica del Cotopaxi ocurrida en el año 1877 fue del tipo explosiva (Mothes, 2004), lo cual conduce a plantear que el volumen de agua sería liberado de forma súbita.

Se plantea que el hidrograma de inicio presenta una forma triangular desfasada, con un pico o valor máximo de crecida y atenuación lenta de manera análoga a los hidrogramas propuestos para el Mount Saint Helens (Brugman \& Meier, 1981) y Nevado del Ruíz (Pierson et al., 1990). Se reporta que el evento eruptivo tendría una duración total de 30 minutos (Wolf, 1878), el cual es considerado en la definición de las condiciones iniciales de contorno del modelo numérico.

De esta manera se plantea en la presente investigación que el pico de crecida ocurra en el primer tercio de la duración total del evento, es decir a los 10 minutos del inicio. Se considera que el volumen del agua es función directa del área o superficie del glaciar multiplicada por el espesor o altura de derretimiento, la cual no es generalizada y varía en función del escenario estudiado (CIERHI, 2017).

Considerando que el agua en estado sólido tiende a variar su volumen, se estima un factor de conversión de 0,9 al cambiar al estado líquido. Además, según la reología del lahar se plantea que la mezcla se componga de un tercio de agua y dos tercios de material sólido (volumen de lahar producido será tres veces mayor al volumen de agua). Para el escenario de calibración del flanco Sur del volcán, de los 10 millones de metros cuadrados $\left(\mathrm{m}^{2}\right)$ de glaciar correspondientes a un volumen total de 40 millones de metros cúbicos $\left(\mathrm{m}^{3}\right)$, alrededor de 36 millones de $\mathrm{m}^{3}$ constituyen el volumen de agua y 108 millones de $\mathrm{m}^{3}$ representan el volumen de lahar. $\mathrm{Al}$ relacionar los volúmenes de lahar con sus correspondientes hidrogramas se obtienen los caudales para cada uno de los cuatros tramos o cauces iniciales de la red de drenaje Sur.

Tabla 3. Caudales máximos para la calibración del modelo numérico

\begin{tabular}{cccc}
\hline Tramo & $\begin{array}{c}\text { Longitud } \\
(\mathbf{k m})\end{array}$ & $\begin{array}{c}\text { Pendiente } \\
(\mathbf{m} / \mathbf{m})\end{array}$ & $\begin{array}{c}\text { Caudal máximo } \\
\left(\mathbf{m}^{\mathbf{3}} / \mathbf{s}\right)\end{array}$ \\
\hline Cutuchi 1 & 14,4 & 0,02 & 39063 \\
San Lorenzo 1 & 8,4 & 0,01 & 28433 \\
Pucahuaicu 1 & 8,2 & 0,07 & 16842 \\
Aláquez 1 & 27,1 & 0,04 & 35214 \\
& & Total & $\mathbf{1 1 9 5 5 2}$ \\
\hline
\end{tabular}

\subsection{Calibración del modelo numérico unidimensional}

Con base en los vestigios de campo del evento eruptivo histórico de junio de 1877, el cual tiene características similares a un escenario eruptivo tipo 3 (VEI 3-4), se obtiene información acerca de las profundidades de flujo. Estos niveles del flujo son generados mediante la simulación numérica unidimensional empleando la base topográfica digital (Modelo Digital del Terreno MDT) con pixel de tres metros que representa la morfología de la zona de estudio.

En principio se analizan 40 secciones que sirven posteriormente para la calibración de otras 163 secciones transversales aledañas. La distribución espacial en el área de estudio de las secciones transversales utilizadas en la calibración del modelo se muestra en la Figura 5.
Con la modelación numérica del escenario de Calibración, se obtiene los coeficientes de rugosidad ( $n$ de Manning) que permiten alcanzar los niveles de flujo obtenidos en el campo.

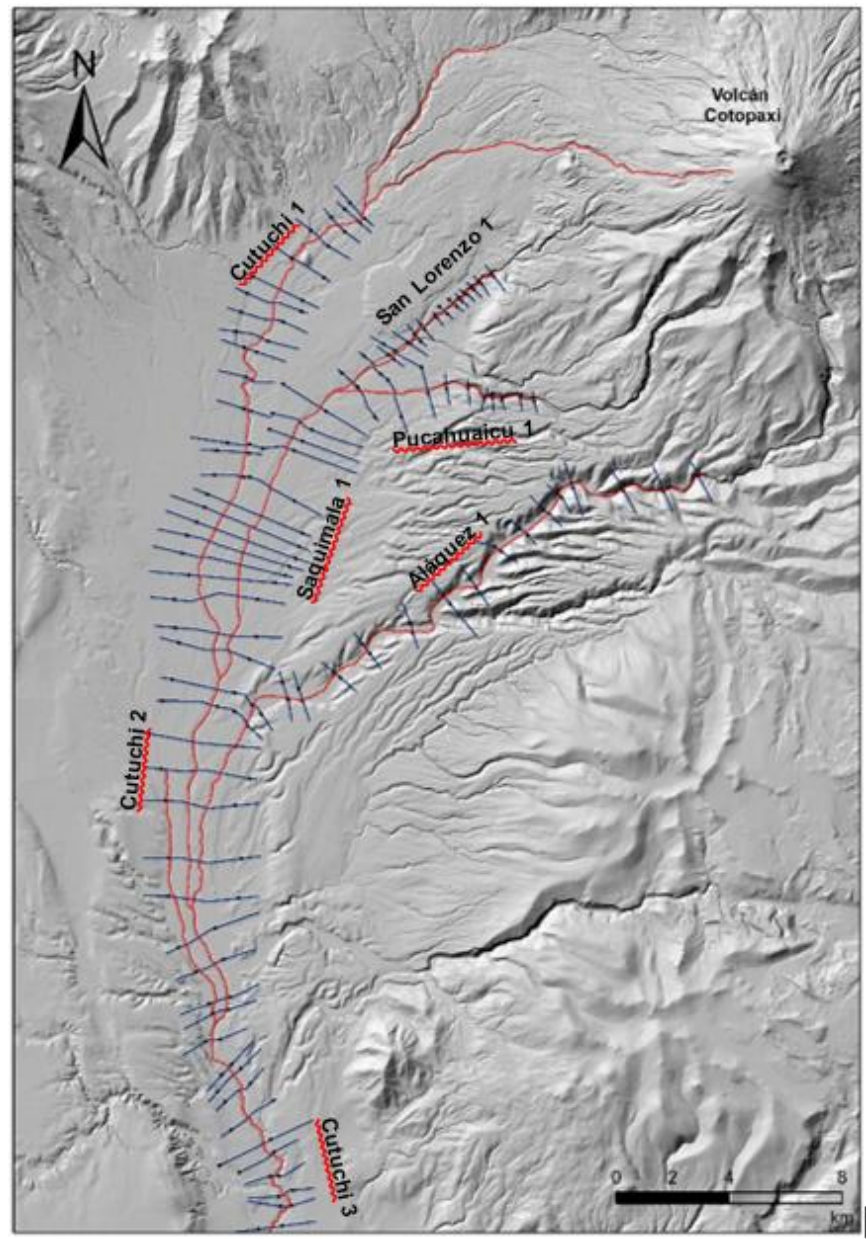

Figura 5. Secciones transversales para la Calibración del modelo numérico

A partir de la simulación numérica de los cauces iniciales se generan hidrogramas de crecida en los tramos intermedios y final del río Cutuchi. De esta manera se representan las características físicas del evento histórico en toda el área de estudio y se obtienen los coeficientes de resistencia al movimiento que se resumen en la Tabla 4.

Tabla 4. Coeficientes de rugosidad del modelo numérico calibrado

\begin{tabular}{cc}
\hline Tramo & $\boldsymbol{n}$ de Manning \\
\hline Cutuchi 1 & $0,083-0,159$ \\
San Lorenzo 1 & $0,077-0,135$ \\
Pucahuaicu 1 & 0,108 \\
Aláquez 1 & $0,031-0,082$ \\
Saquimala 1 & 0,083 \\
Cutuchi 2 & $0,044-0,098$ \\
Cutuchi 3 & $0,025-0,148$
\end{tabular}

\section{RESULTADOS DE LA MODELACIÓN NUMÉRICA}

Con el análisis de flujo permanente se determina la profundidad y velocidad de flujo, considerando el caudal total en cada tramo. Para el análisis de flujo no permanente $(\delta t \neq 0)$ se requiere el uso de hidrogramas de crecida, considerando la variable temporal para obtener resultados orientados a la determinación aproximada y referencial del tiempo de llegada del flujo a una determinada sección. 
De acuerdo a lo mencionado en este documento, se cuenta con cuatro escenarios eruptivos que presentan una probabilidad de ocurrencia determinada que está asociada al Índice de explosividad volcánica (VEI).

Para la determinación de los volúmenes de lahar, se requiere conocer las áreas de glaciar y los espesores de derretimiento promedio en cada caso, los mismos que pueden alcanzar hasta 10 metros de profundidad para el escenario 4 (VEI >4) como se muestra en la Tabla 5.

Tabla 5. Volúmenes de lahar en millones de $\mathrm{m}^{3}$ por escenario eruptivo

\begin{tabular}{|c|c|c|c|c|}
\hline Escenario & VEI & $\begin{array}{c}\text { Espesor } \\
(\mathbf{m})\end{array}$ & $\begin{array}{c}\text { Volumen Agua } \\
\left(\text { millones de } \mathbf{m}^{\mathbf{3}}\right)\end{array}$ & $\begin{array}{l}\text { Volumen Lahar } \\
\left(\text { millones de } \mathbf{m}^{3}\right)\end{array}$ \\
\hline E1 & $1-2$ & 1 & 4 & 12 \\
\hline E2 & $2-3$ & 2 & 8 & 24 \\
\hline E3 & $3-4$ & 5 & 20 & 60 \\
\hline E4 & $>4$ & 10 & 40 & 120 \\
\hline
\end{tabular}

Al relacionar los volúmenes de lahar con los tiempos de duración del evento eruptivo se determinan los hidrogramas de crecida y a partir de estos se obtienen los caudales pico o máximos que se alcanzan en el minuto 10 (primer tercio de la duración total) para cada uno de los tramos iniciales.

Tabla 6. Distribución de caudales en $\left(\mathrm{m}^{3} / \mathrm{s}\right)$ por tramo inicial de simulación

\begin{tabular}{cccccc}
\hline Esc. & Cutuchi & S. Lorenzo & Pucahuaicu & Aláquez & Total \\
\hline E1 & 4029 & 3124 & 2683 & 3551 & 13387 \\
E2 & 8059 & 6248 & 5366 & 7101 & 26774 \\
E3 & 20147 & 15620 & 13415 & 17754 & 66936 \\
E4 & 40295 & 31239 & 26830 & 35507 & 133872 \\
\hline
\end{tabular}

Los hidrogramas de crecida que se van generando a partir del análisis de flujo no permanente alcanzan valores máximos en tiempos no necesariamente simultáneos pues el modelo numérico considera variables como la longitud, pendiente del cauce, geometría de las secciones transversales y coeficientes de resistencia al movimiento. Se obtienen los mayores resultados de profundidad en los tramos encañonados, como el Aláquez 1, con valores de hasta 40 metros. Sin embargo se observa la reducción de estos valores en las zonas planas o de inundación, como en el tramo Cutuchi 2, en el cual se registran profundidades en el cauce de hasta 8 metros de profundidad.

Para el escenario más probable (E3), las velocidades máximas promedio alcanzan valores de hasta $28 \mathrm{~m} / \mathrm{s}$. En la Tabla $7 \mathrm{se}$ reporta los tiempos referenciales correspondientes a la entrada y salida respectivamente, de los tramos de análisis del modelo numérico unidimensional para flujo no permanente.

Tabla 7. Tiempos de llegada en minutos de los caudales máximos

\begin{tabular}{ccccccccc}
\hline \multirow{2}{*}{ Tramo } & \multicolumn{2}{c}{ E1 } & \multicolumn{3}{c}{ E2 } & \multicolumn{2}{c}{ E3 } & \multicolumn{2}{c}{ E4 } \\
& Ent. & Sal. & Ent. & Sal. & Ent. & Sal. & Ent. & Sal. \\
\hline Cutuchi 1 & 10 & 86 & 10 & 83 & 10 & 71 & 10 & 57 \\
$\begin{array}{c}\text { San Lorenzo } \\
\text { 1 }\end{array}$ & 10 & 31 & 10 & 27 & 10 & 24 & 10 & 21 \\
$\begin{array}{c}\text { Pucahuaicu } \\
10\end{array}$ & 26 & 10 & 25 & 10 & 22 & 10 & 21 \\
1 & & & & & & & & \\
Aláquez 1 & 10 & 81 & 10 & 66 & 10 & 52 & 10 & 43 \\
Saquimala 1 & 36 & 47 & 26 & 43 & 23 & 37 & 21 & 31 \\
Cutuchi 2 & 47 & 65 & 43 & 62 & 37 & 55 & 31 & 48 \\
Cutuchi 3 & 65 & 206 & 63 & 175 & 54 & 155 & 47 & 144 \\
\hline
\end{tabular}

Los tiempos de ocurrencia (llegada) de los caudales máximos (pico) en los hidrogramas a la entrada y salida de cada uno de los tramos de simulación varían en función de cada escenario.
Con la modelación numérica se determinan resultados de entre 206 minutos (03:26 horas) y 144 minutos (02:24 horas) correspondientes al tiempo en que el flujo de lahar alcanzaría la salida del tramo Cutuchi 3 para los escenarios 1 y 4 respectivamente.

En la Tabla 8 se presenta los tiempos referenciales asociados a caudales máximos de crecida en varios sitios representativos donde se encuentran zonas pobladas de importancia dentro del área de estudio.

Tabla 8. Tiempos de llegada en minutos de los caudales máximos a varias poblaciones dentro del área de estudio

\begin{tabular}{ccccc}
\hline Población & E1 & E2 & E3 & E4 \\
\hline Aláquez & 60 & 50 & 40 & 30 \\
Latacunga & 95 & 74 & 64 & 55 \\
Salcedo & 120 & 102 & 94 & 82 \\
\hline
\end{tabular}

Los resultados obtenidos en relación a los tiempos de llegada de los flujos de lahar a los sitios representativos de la zona analizada, presentan una variación que guarda relación con la distancia entre el volcán y el lugar de llegada, pero fundamentalmente con el escenario eruptivo. Se observa que conforme se incrementa el escenario eruptivo, la llegada del flujo ocurre en menor tiempo. Para el escenario E3, de acuerdo a lo reportado, se espera que el flujo de lahar alcance la ciudad de Latacunga en aproximadamente 64 minutos.

\subsection{Mapas de afectación por el tránsito de lahares primarios}

En la Figura 6, se presenta en forma gráfica el área de afectación generada para el escenario de mayor probabilidad de ocurrencia (color celeste) y el Mapa de peligros Cotopaxi Sur IG-EPN (color verde). La primera está en función del modelo numérico para flujo no permanente en base a hidrogramas de crecida de las condiciones actuales del glaciar, mientras que la planteada por el IG-EPN (2016), corresponde al levantamiento de vestigios de campo asociados al evento eruptivo histórico del año 1877 con reconocimiento de los depósitos de flujos piroclásticos y zonas de caída de ceniza.

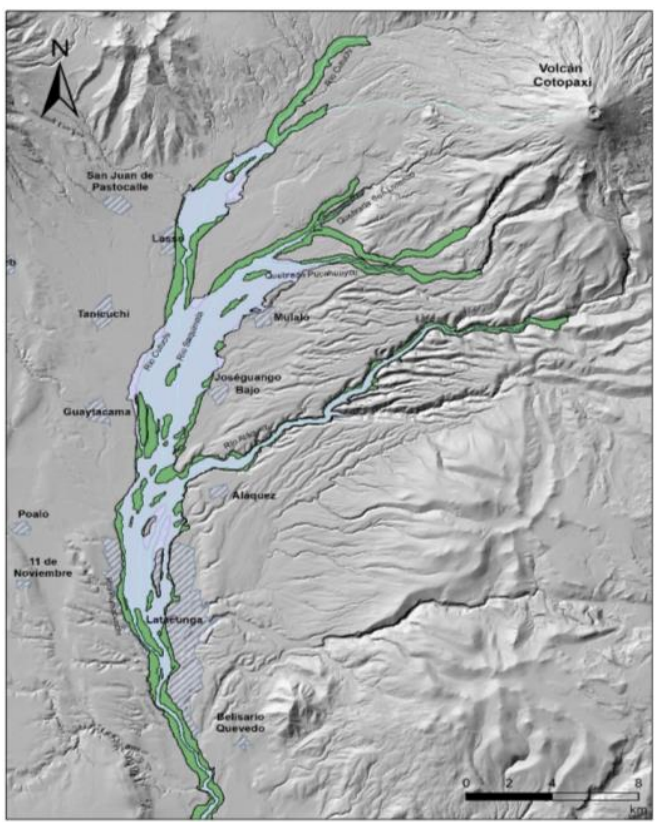

Figura 6. Área de afectación (E3) y Mapa de peligros Cotopaxi Sur (2016) 


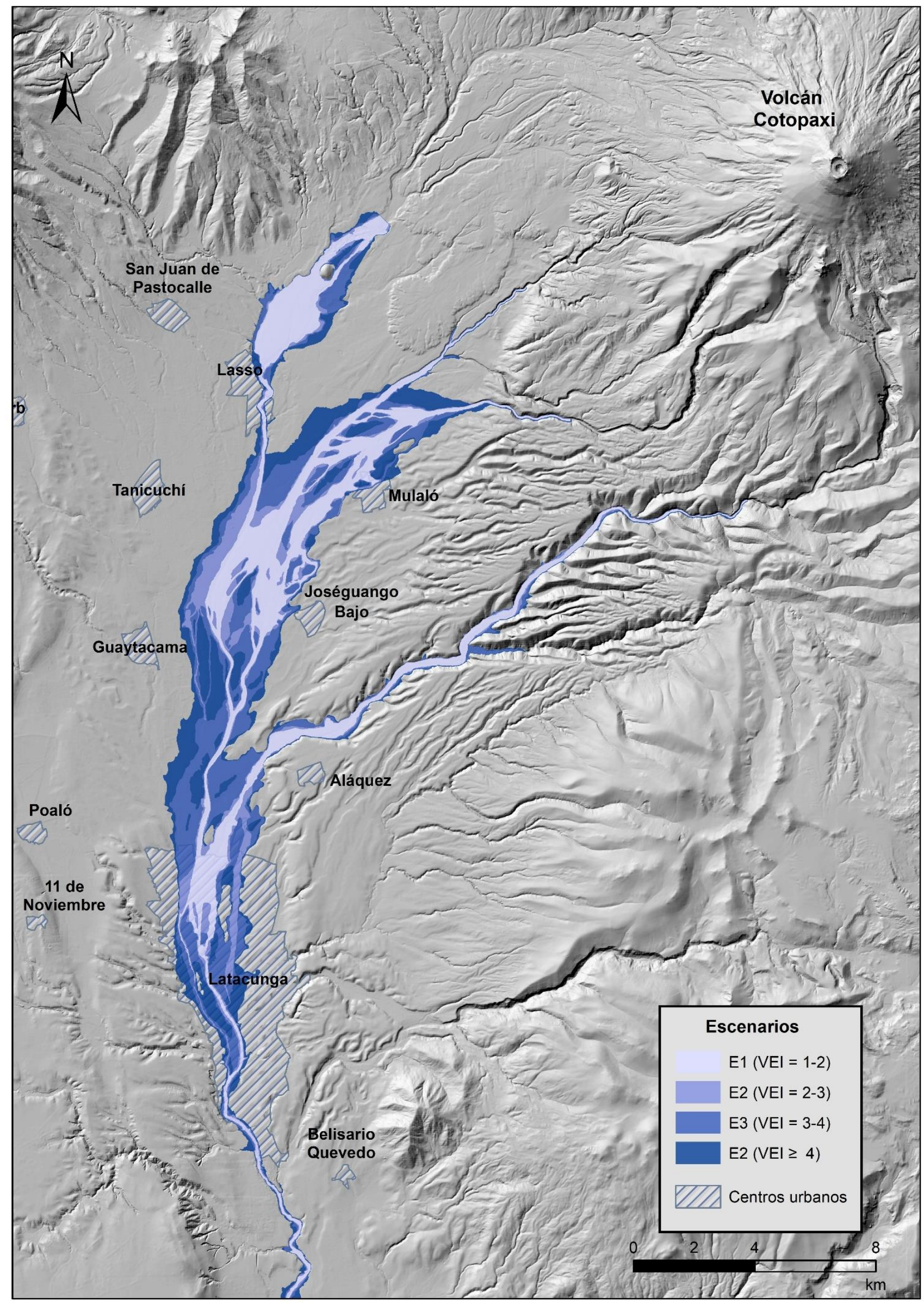

Figura 7. Mapa de afectación por lahares primarios en el flanco Sur-Occidental del volcán Cotopaxi 
Con los resultados de la modelación numérica del flujo de lahares primarios en el drenaje Sur-Occidental del volcán Cotopaxi se elaboran Mapas de afectación considerando los cuatro escenarios eruptivos probables.

En la Figura 7 se presentan las zonas de afectación probables dentro del área estudiada que corresponden principalmente a la ciudad de Latacunga, la cual se encuentra asentada en la zona de expansión natural de tránsito del lahar. Cada uno de los escenarios eruptivos analizados generan zonas de afectación que, de manera general tienden a incrementarse conforme aumenta el escenario analizado.

Es importante recalcar que los resultados obtenidos con la modelación numérica permiten establecer zonas de afectación que deben considerarse como aproximadas y si bien no constituyen límites absolutos, son referenciales. Esto debido a la naturaleza del fenómeno natural estudiado en cuanto a su ocurrencia, magnitud, alcance y grado de amenaza.

\section{CONCLUSIONES}

La simulación numérica unidimensional de flujo permanente y no permanente (variable), es analizada en tramos independientes con la finalidad de alcanzar la convergencia y estabilidad del modelo numérico, en ambos casos se han determinado coeficientes de resistencia al movimiento calibrados en base a los niveles de lahar obtenidos con el trabajo de campo realizado por el IG-EPN relacionado con el evento eruptivo ocurrido en el año 1877.

Los lahares primarios del volcán Cotopaxi tienen características reológicas de flujo hiperconcentrado, granular, no cohesivo similar al flujo dilatante que se puede considerar como turbulento cuando se alcanza valores de caudal unitario superiores a $100 \mathrm{~m}^{3} / \mathrm{s} / \mathrm{m}$, esta condición hace posible el estudio de este tipo de mezclas utilizando modelos numéricos que, si bien son sencillos y producen resultados aproximados, permiten disponer de información relativamente precisa.

Se asume un comportamiento del lahar como flujo turbulento utilizando el modelo numérico HEC-RAS el mismo que, no permite el análisis de aspectos complejos como mezclas multifásicas, procesos de arrastre y depósito de sedimentos, pero es útil ya que produce resultados de forma relativamente rápida y sencilla. El programa requiere la modificación de los coeficientes de resistencia al movimiento ( $n$ de Manning) a fin de alcanzar la calibración del modelo numérico, estos valores varían para este caso particular entre 0,025 y 0,159 .

Con los resultados numéricos del escenario de mayor probabilidad de ocurrencia (E3) se determina que la profundidad máxima alcanza 40 metros de altura, la velocidad máxima promedio se encuentra en el orden de $28 \mathrm{~m} / \mathrm{s}$ y el caudal más alto para este escenario es de $20147 \mathrm{~m}^{3} / \mathrm{s}$. Se determinan tiempos referenciales de llegada o paso del lahar por las poblaciones aledañas más relevantes del área considerada para el estudio en base al análisis de los resultados obtenidos con la modelación numérica para flujo no permanente en la cual se utiliza los hidrogramas de crecida tanto a la entrada como a la salida de los tramos de simulación.
Los mapas de afectación por flujo de lahares primarios del drenaje Sur del volcán Cotopaxi constituyen un aporte técnico significativo puesto que pueden ser usados para tomar decisiones acerca de la definición de sitios seguros, rutas de evacuación e incluso la revisión de la planificación territorial, todo esto sin dejar de lado los planes de concientización, recuperación y mitigación ante los efectos producidos por el paso de lahares ante una eventual erupción volcánica.

\section{AGRADECIMIENTOS}

Los autores agradecen al equipo técnico del Centro de Investigaciones y Estudios en Recursos Hídricos CIERHI de la Escuela Politécnica Nacional por brindar las facilidades y apoyo durante la ejecución de la presente investigación.

\section{REFERENCIAS}

Andrade, D., Hall, M., Mothes, P., Troncoso, L., Eissen, J., Samaniego, P., Egred, J., Ramón, P., Rivero, D. y Yepes, H. (2005). “Los peligros volcánicos asociados con el Cotopaxi”. Quito: Corporación Editora Nacional IG-EPN, IRD. (3), 147 p. (Serie Los Peligros Volcánicos en el Ecuador; 3). ISBN 9978-84-398-1.

Barberi, F., Caruso, P., Macedonio, G., Pareschi, M. T. \& Rosi, M. (1992). "Reconstruction and numerical simulation of the lahar of the 1877 eruption of Cotopaxi volcano (Ecuador)". Acta Vulcanologica. Marinelli Volume. Vol. 2. 35-44.

Bernard, B., Battaglia, J., Proaño, A., Hidalgo, S., Vásconez, F., Hernandez, S. \& Ruiz, M. (2016). "Relationship between Volcanic Ash Fallouts and Seismic Tremor: Quantitative Assessment of the 2015 Eruptive Period at Cotopaxi Volcano, Ecuador" Bulletin of Volcanology 78, no. 11. doi:10.1007/s00445-016-1077-5.

Brugman, M. \& Meier, M. (1981). "Response of glaciers to the eruptions of Mount St. Helens”. 1250. 743-756.

Cáceres, B., Ramírez, J., Francou, B., Eissen, J. P., Taupin, J. D., Jordan, E., Ungerechts, L., Maisincho, L., Barba, D., Cadier, E., Bucher, R., Penafiel, A., Samaniego, P. y Mothes, P. (2005). "Determinacion del volumen del casquete de hielo del volcan Cotopaxi”. INAMHI, IRD, IGEPN, INGEOMINAS.

Cáceres, B.; Jordan, E.; Ungerechts, L.; Francou, B. (2006). "Evaluación geométrica del casquete glaciar del volcán Cotopaxi usando fotogrametría digital". INAMHI. Quito.

Cáceres, B. (2015). "Evolución de los glaciares del Cotopaxi entre 19562015”. Quito.

Cáceres, B. (2016). "Dramatical reduction of Cotopaxi Glaciers during the last volcano awakening 2015-2016", Conference: Agu Fall meeting, DOI 10.13140/RG.2.2.17483.80168, San Francisco - Moscone Center.

Cáceres, B. (2017). "Evolución de la cobertura de glaciar sobre el volcán Cotopaxi para los flancos Norte y Sur”. Informe Inédito. 8 páginas.

Castro, M., Mothes, P., Hidalgo, X. y Sánchez, W. (1992). "Uso del modelo HEC-1 para el estudio de los lahares del volcán Cotopaxi”. Revista Politécnica. Volumen XVII. No. 3. Quito.

Carrillo, J. (2015). "Estudio numérico de la delimitación de zonas de potencial riesgo mediante modelación bidimensional en el flanco sur del volcán Cotopaxi”. CIERHI-EPN. Quito.

Chaudhry, M.H. (1993). “Open-Channel Flow”. Prentice-Hall, Inc., Englewood Cliffs.

Chow, V. T. (1959). "Open-channel hydraulics". McGraw-Hill.

CIERHI Centro de Investigaciones y Estudios en Recursos Hídricos. (2017). "Simulación numérica del flujo de lahares primarios hasta los tramos de implantación de las centrales hidroeléctricas San Francisco y Manduriacu". CELEC E.P.-Escuela Politécnica Nacional. Quito.

Costa, J. E. (1988). "Rheologic, geomorphic, and sedimentologic differentiation of water floods, hyper concentrated flows, and debris flows. Flood Geomorphology". John Wiley \& Sons New York. p 113122.

Costa, J. E. (1997). "Hydraulic modeling for lahar hazards at Cascades volcanoes". Environmental and Engineering Geoscience, 3, 21-30.

Coussot, P. \& Meunier, M. (1995). "Recognition, classification and mechanical description of debris flows". Earth-Science reviews. 
Coussot, P. \& Meunier, M. (1996). "Recognition, classification and mechanical description of debris flows". Earth-Science Review, Vol. 40, No 3, p. 209-227.

Coussot, P., Laigle, D., Arattano, M., Deganutti, A. \& Marchi, L. (1998) "Direct determination of rheological characteristics of debris flow". Journal of Hydraulic Engineering, 124, 865-868.

Coussot, P. (2000). "Mudflow rheology and dynamics, Monograph of IAHR". Rotterdam.

Espín, L. (1993). "Simulación numérica del flujo de lahares a lo largo del río Cutuchi, del volcán Cotopaxi”. Tesis de grado. Escuela Politécnica Nacional. Quito.

Ettinger, S., Mothes, P., Paris, R. \& Schilling, S. (2007). "The 1877 lahar deposits on the Eastern flank of Cotopaxi volcano". Géomorphologie: Relief, processus, environnement. $\mathrm{N}^{\circ}$ 3. p. 271-280.

Gaunt, H., Benjamin, B., Hidalgo, S., Proaño, A., Wright, H., Mothes, P., Criollo, E. (2016). "Juvenile magma recognition and eruptive dynamics inferred from the analysis of ash time series: The 2015 reawakening of Cotopaxi volcano". Journal of Volcanology and Geothermal Research.

Hall, M; Mothes, P; Samaniego, P. (2004). "Análisis del riesgo y estudio de prefactibilidad de las obras de mitigación del volcán Cotopaxi”. Quito.

Hall, M.; Mothes, P.; Andrade, D.; Samaniego, P.; Yépez, H. (2004). "Mapa regional de peligros volcánicos potenciales del volcán Cotopaxi - Zona Sur". IG-EPN . Quito.

Hall, M. \& Mothes, P. "The Rhyolitic-andesitic Eruptive History of Cotopaxi Volcano, Ecuador". Bulletin of Volcanology 70, no. 6 (April 2008): 675-702. doi:10.1007/s00445-007-0161-2.

Hamad, K. (2008). “Aplicaciones del modelo Hec-Ras para el análisis del flujo no permanente con superficie libre". EPN. Quito.

HEC-RAS. US Army Corps of Engineers. (2016). "HEC-RAS river analysis system. Hydraulic reference manual”. Hydrologic engineering center. USA.

IG-EPN Instituto Geofísico de la Escuela Politécnica Nacional. (2015). "Informe especial del volcán Cotopaxi N02". IG-EPN. Quito.

IG-EPN Instituto Geofísico de la Escuela Politécnica Nacional. (2016) "Mapa de Peligros Cotopaxi Sur". IG-EPN. Quito.

Iverson, M., Schilling, S. \& Vallance, J. (1998). "Objective delineation of lahar-inundation hazard zones". GSA Bulletin: 110 (8): 972-984 doi: https://doi.org/10.1130/0016-7606

Iverson, R. M. (2003). "The debris-flow rheology myth. In Debris-Row Hazards Mitigation: Mechanics, Prediction, and Assessment”. Ed. D Rickenmann and C.-L. Chen. Rotterdam: Millpress, pp. 303-314

Iverson, R. M., (2005). "Debris-flow mechanics, en Debris Flow Hazards and Related Phenomena". En: Jakob, Matthias, and Hungr, Oldrich, (eds.), Springer-Praxis, Heidelberg, p. 105-134.

Jordan, E. (1983). "Die Vergletscherung Des Cotopaxi - Ecuador. Zeitschrift Für Gletscherkunde und Glazialgeologie". Universitätsverlag Wagner. Innsbruck.

Jordan, E.; Ungerechts, L.; Cáceres B.; Francou, B.; Peñafiel, A.; Francou, B. (2005). "Estimation by photogrammetry of the glacier recession on the Cotopaxi volcano (Ecuador) between 1956 and 1997'. Hydrological Sciences Journal.

Laenen, A. \& Hansen, P. (1988). "Simulation of three lahars in the mount St. Helens area, Washington using a one-dimensional, unsteady-state streamflow model”. U.S. Geological Survey. Portland.

Lima, P. (2004). "Simulación del flujo lahárico no permanente en el flanco sur del volcán Cotopaxi”. Maestría en Ingeniería de los Recursos Hídricos. Mención Diseño Hidráulico. Escuela Politécnica Nacional. Quito.

Macedonio, G. \& Pareschi, M. T. (1992). "Numerical simulation of some lahars from Mount St. Helens". Journal of Volcanology and Geothermal Research, 54, 65-80.

Mothes, P., Minard, H \& Richard, J. (1998). "The enormous Chillos valley lahar: An ash-flow-generated debris flow from Cotopaxi volcano, Ecuador”. Bulletin of Volcanology 59. No. 4: 233-244.

Mothes, P. (2004), Informe "Flujos de lodos asociados con las erupciones del Volcán Cotopaxi”. Departamento de Geofísica, Escuela Politécnica Nacional. Quito. Ecuador

Mothes, P., Hall, M., Andrade, D., Samaniego, P., Pierson, T., Ruiz, A. y Yépez, H. (2004). "Character, stratigraphy and magnitude of historical lahars of Cotopaxi volcano (Ecuador)". IG-EPN. Quito.

Mothes, P. \& Vallance, J. W. (2015). "Lahars at Cotopaxi and Tungurahua Volcanoes, Ecuador: Highlights from stratigraphy and observational records and related downstream hazards". Volcanic Hazard, Risk, and Disaster, pp. 141-168.

Mothes, P., Hall, M., Espín, P., Vásconez, F., Sierra, D., Córdova, M., Santamaría, S., Marrero, J. y Andrade D. (2016). "Reedición y
Actualización del Mapa de Amenazas Del Volcán Cotopaxi - Zona Sur". IG-EPN, IGM

Naudascher, E. y Castro, M. (2000). "Hidráulica de Canales”. LimusaNoriega Editores, México.

Newhall, C. G \& Self, S. (1982). "The Volcanic Explosivity Index (VEI) an estimate of explosive magnitude for historical volcanism". Journal of Geophysical Research: Oceans, 87,1231-1238. http: //dx.doi.org/10.1029/JC087iC02p01231.

Ordoñez, J., Samaniego, P., Mothes, P. y Schilling, S. (2013). "Los lahares en el volcán Cotopaxi: Delimitación de las zonas amenazadas por el desbordamiento e inundaciones de lahares". $15 \mathrm{pp}$.

Pacheco, R. (2006). Seminario Científico: "Caracterización reológica de flujo de lodos y escombros de origen volcánico: Simulación numérica y aplicaciones prácticas”. Escuela Politécnica Nacional.

Pierson, T. C. (1986). "Flow behavior of channelized debris flows, Mount St. Helens, Washington. In Hillslope Processes”. Ed. A. D. Abrahams. Boston: Allen \& Unwin, pp. 269-296.

Pierson, T. C. y Costa, J. E. (1987). “A rheologic classification of subaerial sediment-water flows. In Debris Hows ¡Avalanches: Process, Recognition, and Mitigation”. Ed. J. E. Costa and G. F. Wieczorek. Geological Society of America, pp. 1-12.

Pierson T. C., Janda R. J., Thouret J. C. y Borrero C. A. (1990), "Perturbation and melting of snow and ice by the 13 November 1985 eruption of Nevado del Ruiz, Colombia, and consequent mobilization, flow and deposition of lahars". Journal of Volcanology and Geothermal Research, 41, p. 17-66.

Pierson, T. C. (1991). "Flow and Deposition of Posteruption Hot Lahars on the East Side of Mount Pinatubo". U.S. Geological Survey.

Pierson T.C. \& Waitt R.B. (1999). "Hydrologic consequences of hotrock/snowpack interactions at Mount St. Helens volcano”. Washington, 1882-84. US. Geol. Surv. Prof. Paper 1586, 1-8.

Pierson, T. C. y Major, J. J. (2014). "Hydrogeomorphic effects of explosive volcanic eruptions on drainage basins". Ann. Rev. Earth Planet. 469507.

Pistolesi, M. (2011). "Physical volcanology of the post-twelfth-century activity at Cotopaxi volcano, Ecuador: Behavior of an andesitic central volcano". GSA Bulletin, V. 123, no. 5/6, p 1193-1215.

Pistolesi, M., Cioni, R., Rosi, M. \& Aguilera, E. (2014). "Lahar hazard assessment in the southern drainage system of Cotopaxi volcano, Ecuador: Results from multiscale lahar simulations". Geomorphology. 207. 51-63. 10.1016/j.geomorph.2013.10.026.

Ramón, P., Vallejo, S., Almeida, M., Cáceres, B. y Gómez, J. C. (2016). "Cotopaxi Volcano awakening in 2015 and increased melting of the glaciers". Cities on Volcanoes 9. Puerto Varas Chile.

Robalino, E. (2006). "Caracterización reológica del flujo lahárico en el Flanco Norte del volcán Cotopaxi”. Maestría en Ingeniería de los Recursos Hídricos. Mención Diseño Hidráulico. Facultad de Ingeniería Civil y Ambiental. Escuela Politécnica Nacional. Quito.

Samaniego, P. (2004), Informe "Escenarios eruptivos en caso de una potencial erupción del Volcán Cotopaxi”. Departamento de Geofísica, Escuela Politécnica Nacional. Quito. Ecuador

Samaniego, P., Mothes, P. y Hall, M. (2004). "Estudio de evaluación de impacto sobre la infraestructura de la EMAAP-Q y la factibilidad de las obras de mitigación ante una eventual erupción del Volcán Cotopaxi”. Quito. Ecuador.

Samaniego, P., Mothes, P., Hall, M., Yepes, H., Castro, M. \& Aguilar, J. (2004). "LAHARZ hazard maps for the Northern drainage of Cotopaxi volcano, Ecuador". IAVCEI General Assembly, Pucon, Chile.

Sánchez, W. A. (1993). "Modelación numérica del tránsito de lahares del volcán Cotopaxi utilizando los programas HEC-1 y DAMBRK". Tesis de grado. Escuela Politécnica Nacional. Quito. 80 p.

Schilling, S. P. (1998). "LAHARZ: GIS Programs for Automated Mapping of Lahar-Inundation Hazard Zones". United States Geological Survey Open-File Report 98-638

Sodiro, L. (1877). "Relación sobre la erupción del Cotopaxi acaecida el día 26 de Junio de 1877”. Imprenta Nacional. Quito. 44 pp.

Takahashi, T. (1991). "Debris Flow”. Monograph of IAHR. Rotterdam.

Thouret, J. C. (1990). "Effects of the november 13, 1985 eruption on the snow pack ans ice cap of Nevado del Ruiz volcano, Colombia". Journal of Volcanology and Geothermal Research, 41, 177-201.

Vera, P. (2017). "Definición de mapas de afectación por flujo de lahares primarios, mediante el uso de modelación numérica”. Maestría en Recursos Hídricos. Mención en Diseño de Obras Hidráulicas. Facultad de Ingeniería Civil y Ambiental. Escuela Politécnica Nacional. Quito. $166 \mathrm{p}$ 
Williams, R. (2006). "Modeling lahars using TITAN2D for the Southern drainage of volcán Cotopaxi: Impact on the city of Latacunga". Master of Science. Department of Geology. The State University of New York at Buffalo.

Wolf, T. (1878). "Memoria sobre el Cotopaxi y su última erupción acaecida el 26 de Junio de 1877”. Imprenta del Comercio. Guayaquil. 45pp.

Yepes, H., Falorni, G., Navas, O. y Andrade, D. (2006). “Cotopaxi Volcano, Lahars on the souhtern drainage of Cotopaxi”. Cuarta conferencia, Ciudades y volcanes. Quito. Ecuador.

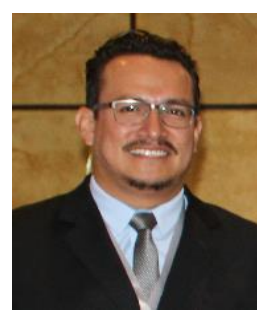

Pablo Alberto Vera Romero (1981). Ingeniero Civil y Magister en Recursos Hídricos mención en Diseño de proyectos hidráulicos de la Escuela Politécnica Nacional. Profesor Ocasional a tiempo completo de la Facultad de Ingeniería Civil y Ambiental de la EPN en el área de Hidráulica. Actualmente es integrante del equipo técnico del Centro de Investigaciones y Estudios en Recursos Hídricos CIERHI-EPN, desarrollando proyectos de investigación básica y aplicada. Miembro la Red de jóvenes investigadores y Editor asociado en la revista Hidrolatinoamericana de jóvenes investigadores y profesionales IAHR.

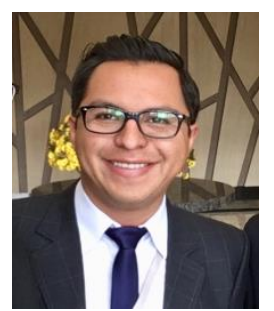

Patricio Rubén Ortega Lara (1986). Ingeniero Civil especialidad Hidráulica de la Universidad Central de Ecuador. Magister en Recursos Hídricos mención Diseño de Proyectos Hidráulicos en la Escuela Politécnica Nacional. Profesor Titular Tiempo Completo desde 2015 en la Facultad de Ingeniería Civil y Ambiental de la EPN. Coordinador del Centro de Investigaciones y Estudios en Recursos Hídricos CIERHIEPN. Presidente de la Red de jóvenes Investigadores de la IAHR. Editor Asociado de la Revista Hidrolatinoamericana de Jóvenes Investigadores y profesionales. Investigador acreditado por el SENESCYT, actualmente cursando sus estudios de Doctorado en la Universidad Politécnica de Cartagena, España.

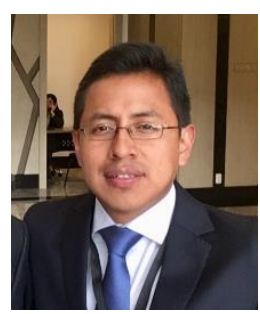

Edwin Patricio Casa Tipán (1988). Ingeniero Civil de la Universidad Central del Ecuador. Magister en Recursos Hídricos mención "Diseño de Proyectos Hidráulicos", Escuela Politécnica Nacional, EPN, Quito. Profesor Ocasional a tiempo completo en la Facultad de Ingeniería Civil y Ambiental de la EPN. Realiza estudios de investigación en el Laboratorio del Centro de Investigaciones y Estudios en Recursos Hídricos, CIERHI de la EPN, sobre temas de diseño, evaluación y optimización de estructuras hidráulicas con base en la modelación física-hidráulica y modelación numérica computacional CFD.

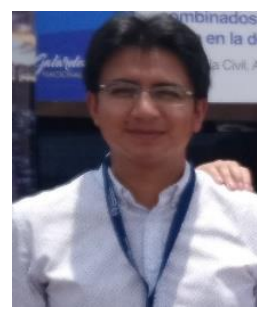

Jorge Andrés Santamaría Chamorro (1992). Ingeniero Civil con mención en Hidráulica graduado en la Escuela Politécnica Nacional. Investigador Asistente en proyectos relacionados con la modelación física y numérica de fenómenos hidráulicos desarrollados en el Centro de Investigaciones y Estudios en Recursos Hídricos CIERHI-EPN. Ganador del Galardón Nacional 2017 de Investigación en el Área de Ingeniería Civil y Vice-Presidente de la Red de jóvenes Investigadores de la IAHR. Actualmente se encuentra cursando sus estudios de Maestría en Hidráulica de la EPN.

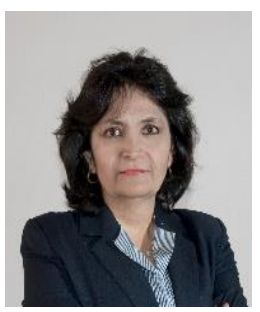

Ximena Hidalgo Bustamante (1960). Ingeniera Civil especialidad Hidráulica de la Escuela Politécnica Nacional, Master of Science en Diseño de Estructuras Hidráulicas y Modelos Hidráulicos en el Instituto de Hidromecánica de la Universidad de Karlsruhe, Alemania. Actual Decana y Profesora titular a tiempo completo de la Facultad de Ingeniería Civil y Ambiental de la EPN desde 1988. Directora del Centro de Investigaciones y Estudios en Recursos Hídricos CIERHI-EPN. Investigadora reconocida en el área de modelación física de estructuras hidráulicas, diseño y optimización de proyectos desarrollados en Ecuador. 